\title{
AUDIT
}

\section{Diagnosis of malignant melanoma by general practitioners and hospital specialists}

\author{
A M Jackson, D R Morgan, R Ellison
}

\begin{abstract}
The aim of this study was to audit all malignant melanomas confirmed histologically in the Scarborough Health District over six years, prompted by the continuing rise in incidence rate nationally and relatively high number of malignant melanomas excised by general practitioners (GPs) in this area. A total of 157 malignant melanomas were diagnosed (60\% from females and $40 \%$ from males) over the six years; primary excisions being carried out by GPs (37\%) and hospital specialists $(63 \%)$. The clinical diagnosis of malignant melanoma was made in $9 \%$ of GP cases and $35 \%$ of the hospital specialist cases. However another $45.5 \%$ of GP cases, and $38 \%$ of hospital specialist cases were regarded as suspicious pigmented lesions clinically. The histological diagnosis was of superficial spreading malignant melanoma in $72 \%$ of the GP and $69 \%$ of the hospital specialist cases. Most of the GP melanomas were excised with a lateral margin of $2 \mathrm{~mm}$ or less (71\%); around half of the hospital excisions had a margin of over $2 \mathrm{~mm}$ (49\%). Most melanomas were 2 mm or less in depth (Breslow depth) in both the GP $(81 \%)$ and hospital specialist $(75 \%)$ series. Over the six year period (1993-98) the incidence of malignant melanomas has continued to rise, but Breslow depth at diagnosis has not changed significantly. It is therefore important to continue with early recognition of this condition by GPs in the first instance, reduction in its incidence being the long term goal. During five years of the study there were only 67 lesions thought clinically to be malignant melanoma (26 GP and 41 hospital specialist cases), but which proved to be benign histologically.
\end{abstract}

(Postgrad Med f 2000;76:295-298)

Keywords: malignant melanoma; histology; pigmented lesion

A M Jackson

D R Morgan

Department of Clinical Audit, Scarborough

Hospital

R Ellison

Correspondence to: Dr Jackson

Submitted 21 June 1999 Accepted 10 September 1999

The incidence of malignant melanoma is increasing steadily in Yorkshire as it is in the whole of the UK. ${ }^{1}$ The Health of the Nation white paper target was to halt the year-on-year increase in the incidence of skin cancer by 2005; more recently, in the white paper Saving Lives: Our Healthier Nation, there has been a single cancer target, to reduce the death rate from cancer among people aged under 75 years by at least a fifth by 2010 from a baseline at $1996 .^{2}{ }^{3}$ The prognosis of malignant melanoma is linked to the Breslow thickness of the tumour at presentation. ${ }^{4}$ This is histologically measured in millimetres. There is an imperative therefore to detect malignant melanoma early when the tumour is likely to be thin. General practitioners (GPs) are at the forefront of the detection process, and in Scarborough a relatively high proportion of malignant melanomas (almost two fifths) are excised by GPs. Accordingly it was decided to audit all malignant melanoma diagnosed histologically in the Scarborough Health District to monitor the incidence, type and Breslow thickness of malignant melanoma, and to compare the diagnosis of this condition by GP with hospital specialists (dermatologists and surgeons). This report analyses the first six years of the audit.

\section{Method}

The pathology department at Scarborough Hospital receives all specimens for histopathological examination from the Scarborough district covering a population of around 220000 . Of approximately 9000 annual requests, some 2000 are skin biopsies; up to 1000 may be pigmented lesions, mostly naevi or seborrhoeic keratoses. From 1 July 1992 to 30 June 1998 all histologically diagnosed malignant melanomas have been audited, by review of clinical notes, for source of request (GP or hospital specialist, that is dermatologist or surgeon), clinical history and diagnosis, age and sex of patient, site of lesion, histological type of malignant melanoma, depth of invasion (Breslow thickness), lateral excision margin, and whether or not a wider excision was performed.

As an adjunct to the above we were also able to perform a study of skin biopsy specimens received over the last five years (1 July 1993 to 30 June 1998) diagnosed clinically as malignant melanoma or suspicious pigmented lesion, but which proved on histological assessment to be benign.

\section{Results}

There were 157 malignant melanomas diagnosed in 156 patients (one patient having two synchronous malignant melanomas) during the six years of the study. The primary excision was carried out by GPs in 58 cases (37\%), and by hospital specialists in 99 cases (63\%) (dermatologists $38 \%$ and surgeons 25\%) 
Table 1 Who performed primary excision 1992-98 for malignant melanomas $(n=157)$; results are number (\%)

\begin{tabular}{lrlr}
\hline & & \multicolumn{2}{l}{ Hospital specialist } \\
\cline { 3 - 4 } Year & \multicolumn{1}{l}{$G P$} & Surgeon & Dermatologist \\
\hline $1992-93$ & $4(18)$ & $8(36)$ & $10(46)$ \\
$1993-94$ & $13(46)$ & $6(22)$ & $9(32)$ \\
$1994-95$ & $5(26)$ & $6(32)$ & $8(42)$ \\
$1995-96$ & $15(48)$ & $5(16)$ & $11(36)$ \\
$1996-97$ & $12(38)$ & $9(28)$ & $11(34)$ \\
$1997-98$ & $9(36)$ & $5(20)$ & $11(44)$ \\
Total & $58(37)$ & $39(25)$ & $60(38)$ \\
\hline
\end{tabular}

Table 2 Clinical diagnosis for primary excision 1992-98 for malignant melanomas $\left(n=146^{\star}\right)$; results are number (\%)

\begin{tabular}{lcl}
\hline Clinical diagnosis & $G P$ & $\begin{array}{l}\text { Hospital } \\
\text { specialist }\end{array}$ \\
\hline Malignant melanoma & $5(9)$ & $32(35)$ \\
Suspicious/pigmented lesion & $25(45)$ & $35(38)$ \\
Mole & $22(40)$ & $7(8)$ \\
Other diagnosis & $3(6)$ & $17(19)$ \\
Total & 55 & 91
\end{tabular}

${ }^{\star}$ Clinical diagnosis not given in 11 cases (three GPs and eight hospital specialists).

Table 3 Histological diagnosis for primary excision 1992 98 for malignant melanomas $(n=157)$; results are number (\%)

\begin{tabular}{lcc}
\hline Histological type & $G P$ & $\begin{array}{l}\text { Hospital } \\
\text { specialist }\end{array}$ \\
\hline Superficial spreading & $42(72)$ & $68(69)$ \\
Nodular & $14(24)$ & $13(13)$ \\
Lentigo maligna melanoma & $2(4)$ & $13(13)$ \\
Acral lentiginous & 0 & $4(4)$ \\
Spindle cell & 0 & $1(1)$ \\
Total & 58 & 99 \\
\hline
\end{tabular}

(table 1$)$. Sixty three $(40 \%)$ patients were male; $93(60 \%)$ were female. The clinical diagnosis was malignant melanoma in five $(9 \%)$ of the GP cases and $32(35 \%)$ of the hospital specialist cases (table 2). In $25(45.5 \%)$ of the GP cases and $35(38 \%)$ of the hospital specialist cases the diagnosis was of suspicious pigmented lesion. A mole was diagnosed clinically in $22(40 \%)$ of the GP cases and seven $(8 \%)$ of the hospital specialist cases. Haemangioma or other diagnosis accounted for the remainder (three GP (5.5\%) and 17 hospital specialist $(19 \%))$.

Histological examination showed a high percentage to be superficial spreading malignant melanomas ( $72 \%$ GP and $69 \%$ hospital specialist), accounting for 110 malignant melanomas (table 3). Nodular malignant melanomas accounted for 14 GP cases (24\%) and 13 hospital specialist cases (13\%). The remainder were lentigo maligna melanoma (two (4\%) GP and 13 (13\%) hospital specialist), acral lentiginous malignant melanoma (no GP and four (4\%) hospital specialist), or spindle cell malignant melanoma (no GP and one (1\%) hospital specialist).

The lateral excision margins were $2 \mathrm{~mm}$ or less in $41(71 \%)$ GP cases and $50(51 \%)$ of hospital specialist cases (table 4). A wider excision was performed in $89(64 \%)$ of the 140 cases $(89 \%)$ where this information could be ascertained. Forty (29\%) of these were GP primary excisions. There was no histological evidence of residual melanoma in any of the wider excision specimens.
Fifty one (36\%) were recorded as having had no wider excision; $11(8 \%)$ being GP primary excisions and $40(28 \%)$ being hospital specialist primary excisions. There was no information about such excision in a further 17 cases (seven GP and 10 hospital specialist). Of the total 68 cases where no wider excision could be confirmed, $24(35 \%)$ had a primary excision margin of $2 \mathrm{~mm}$ or less; 10 were GP cases and 14 were hospital specialist cases. Most (53, or $78 \%$ ) had a Breslow depth of 2 $\mathrm{mm}$ or less.

The Breslow depth was available in 152 cases; the remaining five being biopsies only. Three of the biopsies were nasal melanomas which could not be excised; in one case only the wide re-excision biopsy was received; the remaining case was referred elsewhere for plastic surgery. Where the depth was available 37 (64\%) GP cases and 43 (46\%) hospital specialist cases were less than $1 \mathrm{~mm}$ (table 5). Ten (17\%) GP cases and 27 (29\%) hospital specialist cases were 1-2 mm. Ten (17\%) GP cases and $19(20 \%)$ hospital specialist cases were $2.01-6 \mathrm{~mm}$. The remainder were more than $6 \mathrm{~mm}$ : one GP (2\%) and four hospital specialist $(4 \%)$.

Almost half of the patients were under 50 years of age (74 or $47 \%)$. Almost three fifths (91 or $59 \%$ ) of the malignant melanomas involved face, front and back of chest, arm or hand, the remainder involving abdomen or leg.

There were 63 patients (with 67 lesions) in the five year period, in whom the clinical diagnosis was of the malignant melanomas or suspicious pigmented lesion, but the histological diagnosis was of a benign condition (table 6). Of these, 24 patients (26 lesions) were from GPs and 39 patients (41 lesions) were from hospital specialists. Compound naevi accounted for eight (31\%) of the GP cases and 11 $(27 \%)$ of the hospital specialist cases. Seborrhoeic keratoses accounted for six $(23 \%)$ of the GP cases and three (7\%) of the hospital specialist cases. There were no dysplastic naevi in the GP cases but $10(24 \%)$ in the hospital specialist cases. For the purposes of this study dysplastic naevi were classified as nonmalignant and in the benign category.

Table 4 Primary excision margin 1992-98 for malignant melanomas $(n=157)$; results are number (\%)

\begin{tabular}{lll}
\hline Excision margin $(\mathrm{mm})$ & $G P$ & Hospital specialist \\
\hline$<1.0$ & $11(19)$ & $24(24)$ \\
$1.0-2.0$ & $30(52)$ & $26(26)$ \\
$2.1-3.0$ & $8(14)$ & $13(13)$ \\
$>3.0$ & $9(15)$ & $36(36)$ \\
Total & 58 & 99 \\
\hline
\end{tabular}

Table 5 Breslow depth 1992-98 for malignant melanomas $\left(n=152^{\star}\right)$; results are number (\%)

\begin{tabular}{lcc}
\hline Breslow depth (mm) & GP & Hospital specialist \\
\hline$<1.0$ & $37(64)$ & $43(46)$ \\
$1.0-2.0$ & $10(17)$ & $27(29)$ \\
$2.1-3.0$ & $7(12)$ & $8(8)$ \\
$3.1-6.0$ & $3(5)$ & $11(12)$ \\
$>6.0$ & $1(2)$ & $5(5)$ \\
Total & 58 & 94
\end{tabular}

$\star$ Information not available in five cases as these were biopsies only. 
Table 6 Histological diagnoses in cases thought clinically to be malignant melanoma or suspicious pigmented lesion ( $n=67$ cases; 63 patients); results are number (\%)

\begin{tabular}{lcc}
\hline Histological diagnosis & $G P$ & $\begin{array}{l}\text { Hospital } \\
\text { specialist }\end{array}$ \\
\hline Compound naevus & $8(31)$ & $11(27)$ \\
Seborrhoeic keratosis & $6(23)$ & $3(7)$ \\
Dysplastic naevus & 0 & $10(24)$ \\
Haemangiomas & $1(4)$ & $5(12)$ \\
Miscellaneous & $11(42)$ & $12(29)$ \\
Total & 26 & 41 \\
\hline
\end{tabular}

Haemangiomas accounted for only one (4\%) of the GP cases, but five (12\%) of the hospital specialist cases.

\section{Discussion}

The incidence of malignant melanoma in the Scarborough district is known to be significantly raised compared with West Yorkshire, and England, according to Yorkshire Cancer Organisation (now Northern and Yorkshire Cancer Registry and Information Service) figures, long acknowledged to be reliable. ${ }^{1} \mathrm{~A}$ relatively high proportion (almost two fifths) of malignant melanomas are primarily excised by GPs in this district, compared, for example, with south east Scotland where the figure is about $12 \% .^{5}$ For this reason, and to maintain groups of sufficient size for meaningful analysis, the decision was made to compare the two major groups of clinicians, GPs and hospital specialists, rather than to subdivide hospital specialists into surgeons and dermatologists, and compare three groups.

The proportion of male to female patients presenting with malignant melanoma $(2: 3)$ is within the expected range. ${ }^{6}$

This study confirms the findings of other studies that hospital specialists made a clinical diagnosis of malignant melanoma or suspicious pigmented lesion more often $(73 \%)$ than GPs $(54.5 \%)$, although in this study the difference is significantly less pronounced. ${ }^{58}$ Furthermore, hospital specialist cases were all primarily seen by GPs who, having made a diagnosis of malignant melanoma, may have decided on clinical grounds to promptly refer them.

The higher frequency where a "mole" was diagnosed by GPs (40\%) compared with hospital specialists $(8 \%)$ may well also reflect the referral of more clinically obvious cases to hospital specialists with the more equivocal pigmented lesions being dealt with by GPs.

Superficial spreading malignant melanoma was the most commonly diagnosed type in Scarborough as elsewhere ${ }^{58}$ accounting for $72 \%$ in the GP setting and $69 \%$ in the hospital setting. More nodular malignant melanomas were submitted by GPs (24\%) than hospital specialists $(13 \%)$ possibly reflecting relative ease of diagnosis and excision of this relatively uncommon type. In contrast only $4 \%$ of the GP submissions were lentigo maligna melanoma (the invasive form of lentigo maligna), compared with hospital specialists $(13 \%)$, reflecting relative difficulty of excision due to site, rather than recognition, and hence higher referral to hospital of this type.

The lateral excision margins tended to be narrower with GP cases, being mostly $2 \mathrm{~mm}$ or less $(71 \%)$ compared with $51 \%$ of hospital specialist cases, so confirming the findings of others. ${ }^{58}$ However, the margin was less than 1 $\mathrm{mm}$ in $16 \%$ of GP cases and a similar proportion $(18 \%)$ of hospital specialist cases. A wider excision was subsequently performed in 89 of the cases $(64 \%)$ where this information was available. We are aware of several cases where, for reasons of the anatomical site of the primary tumour or general health of the patient, a wider excision was not performed.

It is recommended that primary excision of melanoma should include a minimum lateral clearance of $2 \mathrm{~mm}$ together with a thin cuff of subcutaneous fat. ${ }^{9}{ }^{10}$ Although there is no compelling evidence to date that incomplete primary excision predisposes to metastasis, ${ }^{11}$ provided further re-excision occurs within 30 days, ${ }^{11}{ }^{12}$ this does allow accurate Breslow thickness to be measured and hence determination of width of re-excision.

Most malignant melanomas were $2 \mathrm{~mm}$ depth or less (Breslow thickness) ${ }^{58}$ whether excised by GP $(81 \%)$ or hospital specialist $(75 \%)$. There is therefore no evidence in this as in other studies ${ }^{5}$ that earlier lesions, as judged by this measurement, are being submitted in differing proportions by either group. It is also reassuring that most malignant melanomas are early (thin) at diagnosis. This confirms the findings of other groups and the trend over the last two decades for decreasing median melanoma thickness. ${ }^{13}$ Our findings do however suggest this trend may have reached a plateau.

In our experience only a very small proportion (around 1\%) of all pigmented skin lesions biopsied were thought clinically to be a malignant melanoma or suspicious pigmented lesion, and shown histologically to be benign; GPs were no more likely than hospital specialists to do this.

All malignant melanomas are likely to initially be seen by GPs and each GP will make a decision as to whether to excise or refer to a hospital specialist. We have found no evidence to suggest that GPs in this area are not recognising and managing adequately skin lesions suspicious of malignant melanoma. This is all the more important in view of the high proportion of such lesions excised by GPs in Scarborough and the realisation that higher referral rates of pigmented lesions could overwhelm the hospital system. It is important that all GPs are aware of the clinical features of malignant melanoma. In general, lesions that are not obviously benign, or changing moles, should be seen by GPs and either removed in their entirety for pathological examination, or referred and dealt with by appropriately trained hospital specialists within four weeks. The vast majority of pigmented lesions submitted for histology are correctly diagnosed benign conditions, mostly naevi or seborrhoeic keratoses. Incisional biopsy may occasionally be acceptable, for example in the differential diagnosis of lentigo maligna or acral melanoma. Histological examination is mandatory in all cases.

What is disappointing is the sustained increase in incidence of malignant melanoma 
Box 1: Clinical features suggesting malignant melanoma

- Major signs (change in size, shape, and colour).

- Minor signs (inflammation, crusting or bleeding, sensory change such as itch, diameter $7.0 \mathrm{~mm}$ or more).

- Biopsy whole lesion with full thickness skin biopsy to include $2.0 \mathrm{~mm}$ clear margin.

- Histological examination of all excised pigmented lesions mandatory.

\section{Box 2: Breslow tumour thickness}

- Measured by microscopy.

- Thin (0-1.49 mm) denotes good prognosis, $>90 \%$ five year survival.

- Thick $(>3.50 \mathrm{~mm})$ denotes poor prognosis, $<50 \%$ five year survival.

- Survival with any thickness better for females than males.

over the past six years. This will clearly necessitate continuation of prevention measures especially avoidance of sunburn in children, and limitation of total cumulative sun exposure in all white skinned individuals.

It is intended to continue this local audit of excised malignant melanomas in Scarborough, and recommended that such audit be carried out elsewhere to ensure optimum management whether by GP and/or hospital specialist. Early, accurate diagnosis offers the besthope for reduction in mortality, in advance of the long term goal of a reduction in incidence.

\section{Learning points}

Clinical features, Breslow tumour thickness, and diagnosis of malignant melanoma are shown in boxes 1,2 , and 3 .

The authors are indebted to Miss Tracey Stephenson for typing the manuscript.
Box 3: Diagnosis of malignant

melanoma

- $60 \%$ female: $40 \%$ male.

- Primary excision by GPs in $37 \%$ and hospital specialists in $63 \%$.

- Clinical diagnosis of malignant melanoma or suspicious pigmented lesion in $54.5 \%$ of GP excisions and $73 \%$ of hospital specialist excisions.

- Superficial spreading malignant melanoma commonest type for GP (72\%) and hospital specialist (69\%) excisions.

- Most malignant melanomas $<2.0 \mathrm{~mm}$ thickness whether excised by GPs $(81 \%)$ or hospital specialists (75\%).

- $3 \%$ of all pigmented skin lesions biopsied are malignant melanoma.

- $1 \%$ of all pigmented skin lesions biopsied are thought clinically to be malignant melanoma or suspicious pigmented lesions but are histologically benign.

1 Northern and Yorkshire Cancer Registry Information Service (NYCRIS). Data for health authority boundaries at April 1996. Leeds: NYCRIS, 1997

2 Department of Health. Health of the nation. (White paper.) London: Department of Health, 1992.

3 Department of Health. Saving lives: our healthier nation. (White paper.) London: Department of Health, 1999.

4 Breslow A. Thickness, cross-sectional areas and depth of invasion in the prognosis of malignant melanoma. Ann Surg 1970;172:902-8.

5 Herd RM, Hunter JA, Mclaren KM, et al. Excision biopsy of malignant melanoma by general practitioners in South East Scotland 1982-91. BMF 1992;305:1476-8.

6 Austoker J. Melanoma: prevention and early diagnosis. BMF 1994;308:1682-6.

7 Bricknell MCM. Skin biopsies of pigmented skin lesions performed by general practitioners and hospital specialists. prff Gen Pract 1993;43:199-201.

8 Khorshid SM, Pinney E, Newton-Bishop JA. Melanoma excision by general practitioners in North-East Thames region, England. Br f Dermatology 1998;138:412-17.

9 Urist MM, Balch CM, Milton GW. Surgical management of the primary melanoma. In: Balch CM, Milton GW, eds. Cutaneous melanoma. Philadelphia: Lippincott, 1985: 71-90.

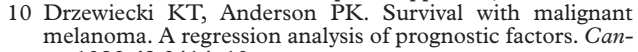
cer 1982;49:2414-19.

11 Lederman J S, Sober AJ. Does biopsy type influence survival in clinical stage 1 cutaneous melanoma? f Am Acad Dermatol 1985;13:983-7.

12 Landthaler M, Braun-Falco O, Leitl A, et al. Excisional biopsy as the first therapeutic procedure versus primary wide excision of malignant melanoma. Cancer 1989;64:1612-6.

3 Williams HC, Smith D, du Vivier AWP. Evaluation of public
education campaigns in cutaneous melanoma: the Kings Coleducation campaigns in cutaneous melanoma: the Kings C
lege Hospital experience. Br f Dermatol 1990;123:85-92. 